\title{
Recent Advances in Molecular Electronics Based on Carbon Nanotubes
}

\author{
Jean-Philippe Bourgoin*, Stéphane Campidelli, Pascale Chenevier, Vincent Derycke, Arianna \\ Filoramo, and Marcelo F. Goffman
}

\begin{abstract}
Carbon nanotubes (CNTs) have exceptional physical properties that make them one of the most promising building blocks for future nanotechnologies. They may in particular play an important role in the development of innovative electronic devices in the fields of flexible electronics, ultra-high sensitivity sensors, high frequency electronics, opto-electronics, energy sources and nano-electromechanical systems (NEMS). Proofs of concept of several high performance devices already exist, usually at the single device level, but there remain many serious scientific issues to be solved before the viability of such routes can be evaluated. In particular, the main concern regards the controlled synthesis and positioning of nanotubes. In our opinion, truly innovative use of these nano-objects will come from: i) the combination of some of their complementary physical properties, such as combining their electrical and mechanical properties, ii) the combination of their properties with additional benefits coming from other molecules grafted on the nanotubes, and iii) the use of chemically- or bio-directed self-assembly processes to allow the efficient combination of several devices into functional arrays or circuits. In this article, we outline the main issues concerning the development of carbon nanotubes based electronics applications and review our recent results in the field.
\end{abstract}

Keywords: Carbon nanotubes, CNTFET, Functionalization, Neuromorphic computing architectures, Selfassembly

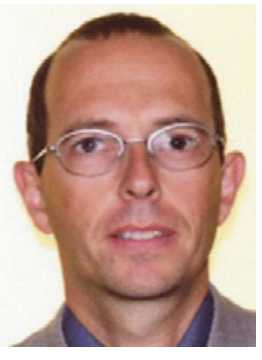

Jean-Philippe Bourgoin is the Director of the Atomic and Alternative Energies Commission (CEA www.cea.fr) Nanoscience program and formerly head of the Laboratoire d'Electronique Moléculaire. He studied at the Ecole Normale Supérieure de Cachan, Aggregation of Physics, $\mathrm{PhD}$ on organic conducting monolayers (1991), habilitation on solidstate physics (2001). A post-doctoral year was spent at the IBM Research Laboratory in Rüschlikon working with Dr H. Rohrer (Nobel Prize) and Dr B. Michel on a microwave STM (1993-94). He has been head of the joint CEA-Motorola Molecular Electronics Laboratory (2001-2003), deputy

\footnotetext{
${ }^{\star}$ Correspondence: Dr J.-P. Bourgoin Laboratoire d'Electronique Moléculaire Service de Physique de l'Etat Condensé (CNRS URA 2464)

CEA, IRAMIS,

91191 Gif sur Yvette, France

Tel.: +3316908 8553

Fax: +3316908 8786

E-mail: jean-philippe.bourgoin@cea.fr
}

manager of the Service de Physique de l'Etat Condensé (2005-2006), co-Head of the CEA research programme on 'Chemistry and Nanoelectronics' (2005-2006). Co-founder and member of the board of the Paris Region Nanosciences Competence Center CnanoIDF (>2800 researchers, 2005-). Expert for various international funding agencies and programs (NSF, ESF, ERC, Canada, Ireland, Netherlands, European Commission) and with the French National Research Agency where he is President of the micro-nano scientific committee. He is presently associate editor of the Journal of Nanoparticle Research. His scientific interests include molecular electronics, carbon nanotubes devices, self-assembly and new architectures of computation. 94 Publications, $>75$ invited conferences, 10 patents.

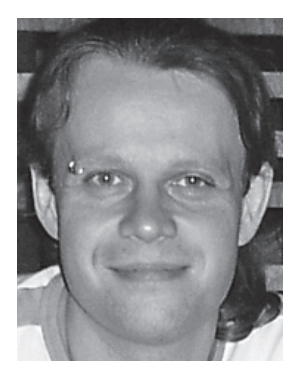

Stéphane Campidelli was born in 1974 in France. He obtained his 'DEA' (MSc degree) in organic chemistry from the University of AixMarseille III (France) in 1998. After a short period in industry, he began his $\mathrm{PhD}$ in 2000 under the supervision of Prof. Robert Deschenaux at the University of Neuchâtel in Switzerland. His $\mathrm{PhD}$ dissertation was awarded the 'prix Syngenta Monthey'. In 2004, he joined the group of Prof. Maurizio Prato at the University of Trieste (Italy) for a post doctoral fellowship. In January 2007 he moved to CEA Saclay where he is currently working as researcher.

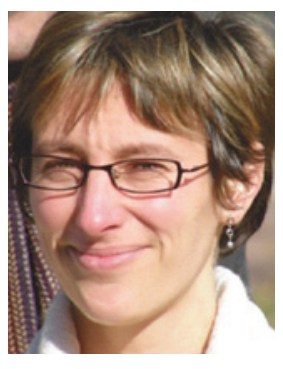

Pascale Chenevier was born in 1974. She completed her degree in chemistry and biology at Ecole Normale Supérieure de Lyon. She worked in teams of very different domains before joining CEA in 2004: protein biochemistry at Univ. Lyon I, molecular and cellular biology, then supramolecular chemistry at ENS Lyon, targeted drug delivery during her PhD at Bordeaux (CRPP lab., PhD in 2001), and finally biophysics at the Cornell University Synchrotron in the USA. She deals now with carbon nanotube chemistry for molecular electronics and energy research: grafting on CNTs for light-driven or high frequency CNT transistors, toxic gas detectors, protein grafting on CNTs as regrowth catalysts, photosensitizer or fuel cell biocatalysts.

Vincent Derycke received his $\mathrm{PhD}$ in physics from the University of Paris XI-Orsay in 2000. His PhD work in surface science was devoted to the study of silicon carbide at the atomic scale. He then joined 


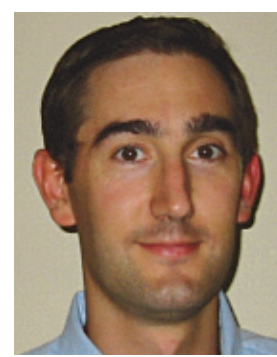

the research group of P. Avouris at IBM Yorktown where he participated in the development of nanotube-based field effect transistors. In 2002, he joined the Molecular Electronics Laboratory of the CEA in Saclay (IRAMIS, SPEC). Since June 2008, he is in charge of this research group. His activity principally focuses on carbon nanotube-based high-frequency and optoelectronic devices and on programmable circuit architectures. He is the author or co-author of 40 publications and 8 patents.

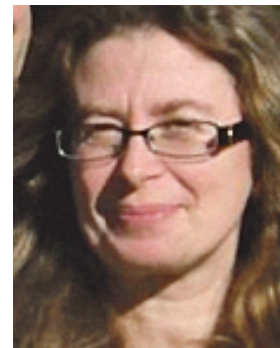

Arianna Filoramo

received her $\mathrm{PhD}$ degree in solid state physics at the ENS (Ecole Normale Superieure) in Paris in 1997 on time-resolved spectroscopy of semiconductor hereostructure (excitonic population dynamics and spin relaxation). In 1998 she joined the Molecular Electronics Research Lab of Motorola. Since 2004 she carries on her activity in the LEM (CEA Saclay). Her main topics concerns molecules, carbon nanotubes (purification, chemistry, characterization and devices fabricated by nanomanipulation) and DNA scaffolds (metallic nanowires and DNA-carbon nanotube linkage).

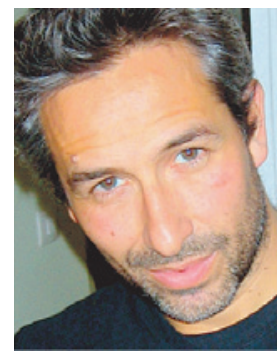

Marcelo Fabián Goffman is a member of the Molecular Electronics Laboratory of CEA since 2001. He obtained his $\mathrm{PhD}$ degree in solid state physics at Instituto Balseiro, Bariloche, Argentine in 1997 working on the properties of vortices in high temperature superconductors. He received, in 1998, an honorary mention of the ' $\mathrm{J} J$ Giambiagi' prize of the Argentinean Academy of Physics for his thesis work. He joined (1998-2000) the Quantronics Group of M. Devoret, D. Esteve and C. Urbina at SPECCEA Saclay (France), where he worked on the superconducting electronic transport through single atoms. Its interests as experimentalist in nano-physics are focused on the understanding of electronic transport in molecular based hybrid circuits and on the development of carbon-nanotube based electromechanical devices.

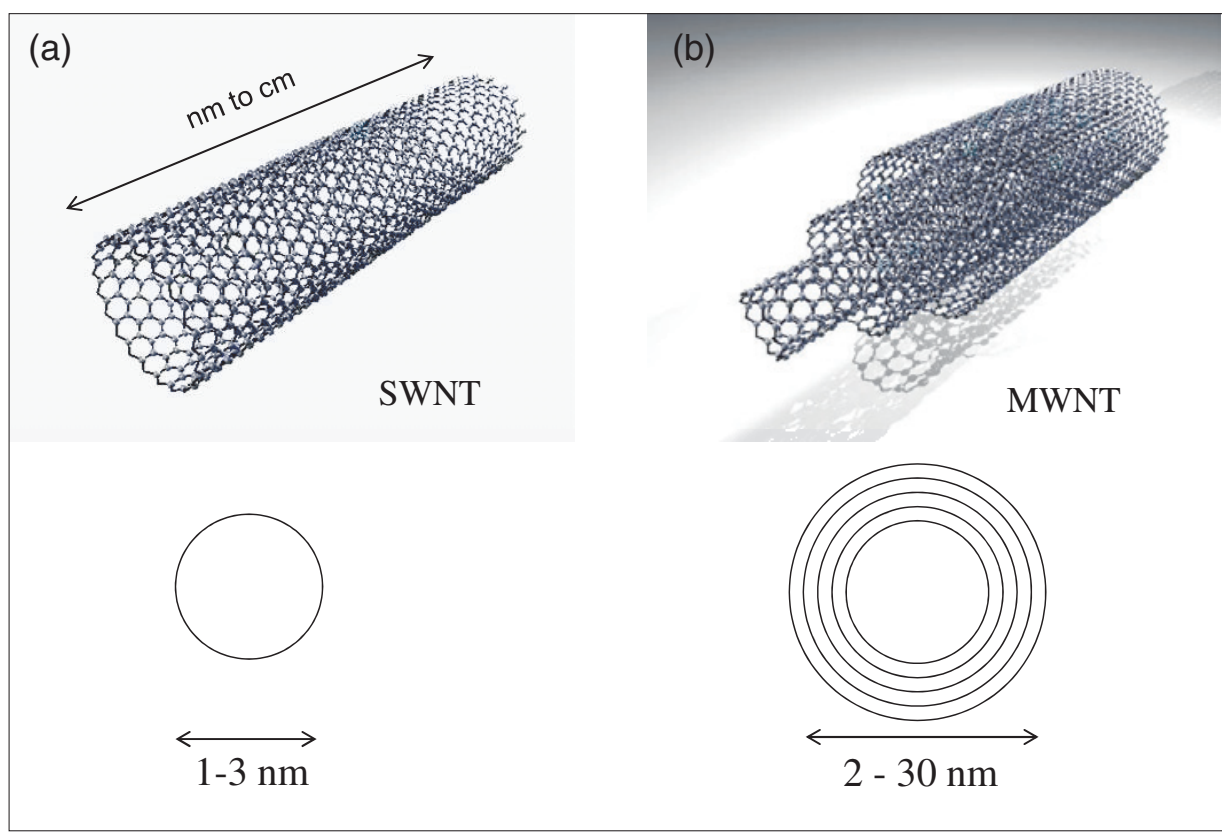

Fig. 1. Schematic view and typical size of (a) a SWNT and (b) a MWNT.

\section{Introduction}

Molecular electronics targets the use of molecules, and, by extension, molecularsized objects for electronics. This domain saw pioneering experiments ten to fifteen years ago, with the first transport measurements on single molecules, on single nanoparticles and on single carbon nanotubes. The reader will find a historical review of this field in ref. [1]. In the present article, we focus on carbon nanotubes which are particularly promising for use in various types of electronic applications and functions.

Carbon nanotubes and fullerenes are different allotropic forms of carbon. Fullerenes are closed-cage molecules containing only carbon atoms disposed in a hexagonal and pentagonal interatomic bonding network. Nanotubes are like large, cylindrical fullerenes with usual aspect ratio of $10^{3}$ to $10^{5}$ (see Fig. 1). More precisely, a single-wall carbon nanotube (SWNT) is a cylinder obtained by rolling-up a graphene sheet of hexagonal carbon rings (with halffullerenes potentially capping the shell ends). Similarly, multi-wall nanotubes (MWNTs) can be schematized as a rolledup stack of graphene sheets in concentric shells (like Russian dolls). It turns out that depending on its geometry and on the way the graphene sheet is rolled-up, the SWNT is either metallic or semi-conducting.

While the different allotropic forms of carbon (diamond, graphite, graphene, $\mathrm{C}_{60}$ molecule etc.) refer to well-defined structures, the term 'carbon nanotube' (CNT) encompasses a large variety of different objects, which differ from each other in terms of diameter, length, chirality, electronic properties and number of shells in the case of MWNT. It can be an opportunity for future applications since different types of nanotubes can be better suited for different types of use. For example, the mechanical properties of multi-wall nanotubes with intermediate diameter $(5-15 \mathrm{~nm})$ are of particular interest in the field of nanoelectromechanical systems (NEMS). Semiconducting SWNTs can serve as channels in field effect transistors, while metallic SWNTs could be important as nanoscale interconnects or nanoscale electrodes.

In the past, a strategy was developed at the Laboratory of Molecular Electronics of the CEA Saclay to build and study electronic devices, starting from commercial nanotubes and going through different steps of chemical purification, ${ }^{[2,3]}$ solubilization, functionalization, ${ }^{[3,4]}$ and assembly..$^{[5-11]}$ We considered these steps as the key to the development of innovative electronic functions. In particular, we studied carbon nanotube field effect transistors and their chemical optimization, ${ }^{[12,13]}$ high frequency nanotube transistors, ${ }^{[14-16]}$ nanotubebased nano-electromechanical systems (NEMS), ${ }^{[17,18]}$ and nanotube-based optoelectronic devices with memory capabilities and related computing architectures. [19-21]

\section{Mastering the Chemistry of CNTs}

Since the very first synthesis of CNTs, the issues of purification, sorting and functionalization have been the focus of a lot of attention by researchers worldwide.

Along with the spreading use of SWNT in various domains, SWNTs functionalization methods flourished to help process this highly insoluble material, as well as to add new functionalities such as tuned light 
absorption or chemical sensing. Among the covalent functionalization methods, diazonium to SWNTs coupling is the most wide-spread because of its handiness and high yield. Nevertheless, the mechanism of this complex reaction remained mysterious. Using chemical kinetics methods, we thoroughly studied the mechanism of the reaction, aimed at mastering the coupling rate and controlling the selectivity of the reaction towards metallic SWNTs. Chemical kinetics as well as electron spin resonance demonstrated without ambiguity a free radical chain reaction involving SWNT stable radicals. ${ }^{[3]}$ In the propagation phase, the aryl radical reacts with SWNT to yield an aryl-SWNT radical, then the aryl-SWNT radical reduces the aryl diazonium into a new aryl radical. The origin of the selectivity of the reaction towards metallic SWNTs was unveiled. Indeed, metallic SWNTs have a higher HOMO (Higher Occupied Molecular Orbital) than semiconducting SWNTs, which enhances their reactivity towards the electrophilic aryl radical.

Nanotubes by themselves already combine many exceptional physical properties. Still, both the development of new functionalities for individual devices and the assembly of complex structures and circuits could be improved by the incorporation of highly engineered molecules on the nanotube surfaces. There is thus a real need for simple and versatile procedures which allow the introduction of new functional groups onto the nanotube surface. In particular, we recently investigated the functionalization of SWNTs with Zn-phthalocyanine derivatives via 'click chemistry' and demonstrated that this concept can be used for the realization of photovoltaic cells. ${ }^{[4]}$ The term 'click chemistry' ${ }^{[22]}$ defines a series of clean, versatile, specific chemical reactions, easy to realize and exhibiting simple purification processes (absence of by-products). Among the large collection of organic reactions, Huisgen cycloaddition (1,3-dipolar cycloaddition between azide and acetylene derivatives in the presence of $\mathrm{Cu}(\mathrm{I})$ catalyst) represents the most effective reaction of the 'click chemistry'. ${ }^{[23-26]}$ It has been demonstrated that the emerging field of 'click chemistry' can bring very elegant solutions to easily achieve nanotube-based functional materials. ${ }^{[4,27,28]}$ In our recent experiments, we described the functionalization of SWNTs with 4-(2-trimethylsilyl) ethynylaniline and the subsequent attachment of a zincphthalocyanine $(\mathrm{ZnPc})$ derivative using the reliable Huisgen 1,3-dipolar cycloaddition (Fig. 2). The SWNT-ZnPc nanoconjugate was fully characterized and a photoinduced communication between the two photoactive components (i.e., SWNT and ZnPc) was identified. Such beneficial features led

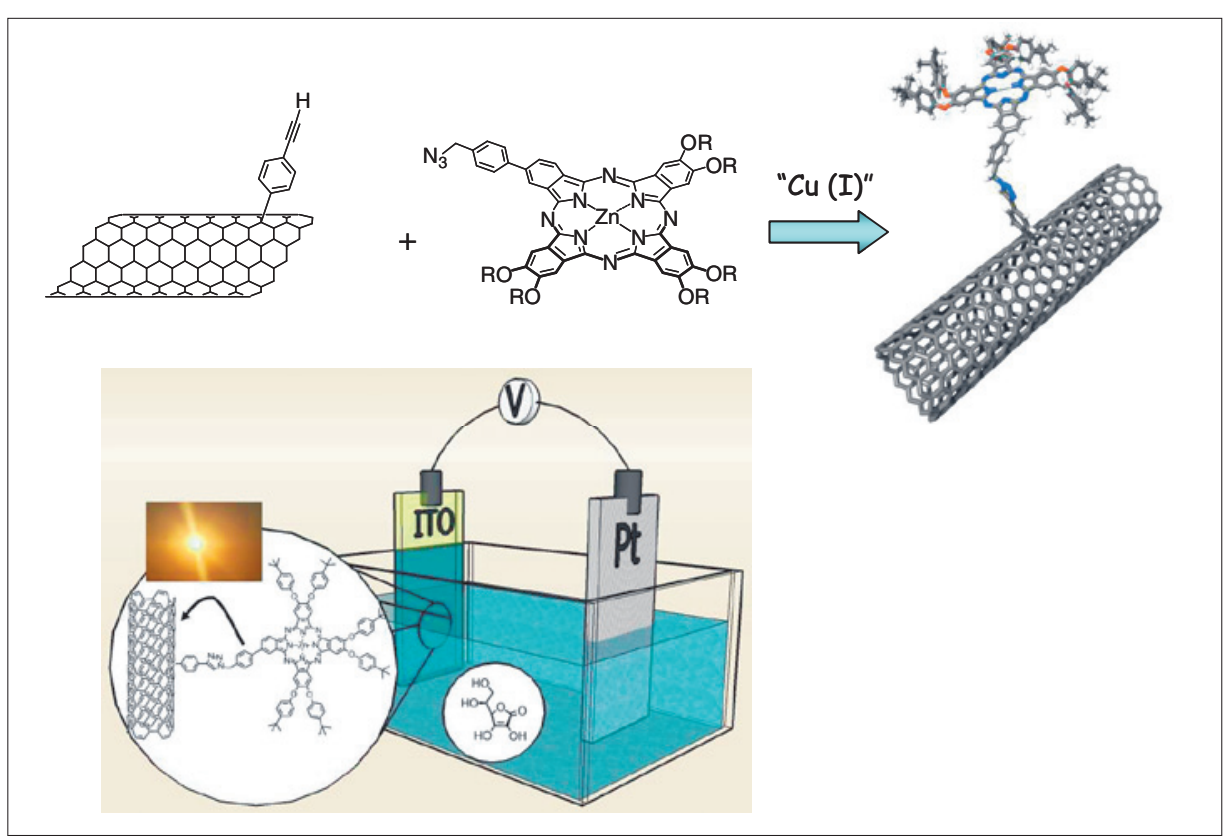

Fig. 2. Principle of the addition of phthalocyanine on SWNTs via 'click chemistry' and schematic representation of the photoelectrochemical cell containing the SWNT-ZnPc conjugate. From ref. [66] after ref. [4].

us to incorporate the SWNT-ZnPc hybrid in a photoelectrochemical cell as photoactive material in an ITO photoanode. The use of such hybrid CNT-molecule objects is also studied in our laboratory in the context of optically controlled nanotube transistors.

\section{Selective Placement of Nanotubes}

To fully take advantage of the unique electrical properties of SWNTs in device/ circuit applications, it is necessary to be able to selectively place them at specific locations on a substrate with a low cost and high yield technique, preferentially based on self-assembly. We developed three different techniques of selective deposition: i) chemically-assisted self-assembly on patterned molecular monolayers, ii) dielectrophoresis, and iii) bio-directed selfassembly.

During the last 10 years, we have developed and optimized a technique of localized nanotube deposition assisted by self-assembled monolayers (SAMs). The approach is based on the grafting of amine-terminated SAMs to modify the surface properties of certain regions of a $\mathrm{SiO}_{2}$ substrate. This affects the interactions between the sidewalls of CNTs and the surface so that the CNTs in solution are preferentially captured on the pattern. This kind of study started with the pioneering works of Lui et al., ${ }^{[29]}$ Muster et al. ${ }^{[30]}$ and Choi et al. in the laboratory. ${ }^{[5]}$ The combination of adapted organic solvent and well organized monolayers allowed us to control both the deposition density and se- lectivity. ${ }^{[6-8]}$ We efficiently used this technique to build field-effect transistors based on individual SWNTs ${ }^{[13,19]}$ or network of $\mathrm{CNTs}^{[14]}$ and electromechanical switches based on individual MWNTs. ${ }^{[18]}$

A second deposition technique, which is of particular interest for carbon nanotubes, is dielectrophoresis (DEP). ${ }^{[31]}$ It consists in depositing a droplet of a nanotube solution on pre-patterned electrodes and applying between these electrodes an $\mathrm{AC}$ electric field in the $\mathrm{MHz}$ range. The polarisability of carbon nanotubes induces an increase in nanotube concentration in the area of high electric field gradients and the favourable alignment of the nanotubes along the field lines. As shown in Fig. 3, the method works for both MWNTs and SWNTs, and for both individual and dense networks of nanotubes. Using dielectrophoresis, such individual MWNT can be incorporated in NEMS, while dense nanotube networks are important for the development of high frequency devices.

Because of its unique recognition properties, its size and the sub-nanometric resolution, DNA is of particular interest for positioning and organizing nanomaterials, and in particular SWNTs. Moreover, in DNA-directed nanoelectronics, DNA can be envisioned not only as a positioning scaffold, but also as a support for the conducting elements. ${ }^{[32,33]}$ With the aim to fabricate carbon nanotube-based devices by combining DNA with SWNTs, we developed a non-covalent approach based on biotin-streptavidin molecular recognition properties $^{[9-11]}$ that preserves the structural quality of the CNTs (Fig. 4). Complex structures can be fabricated by DNA-strand engineering. For example, we incorporated 

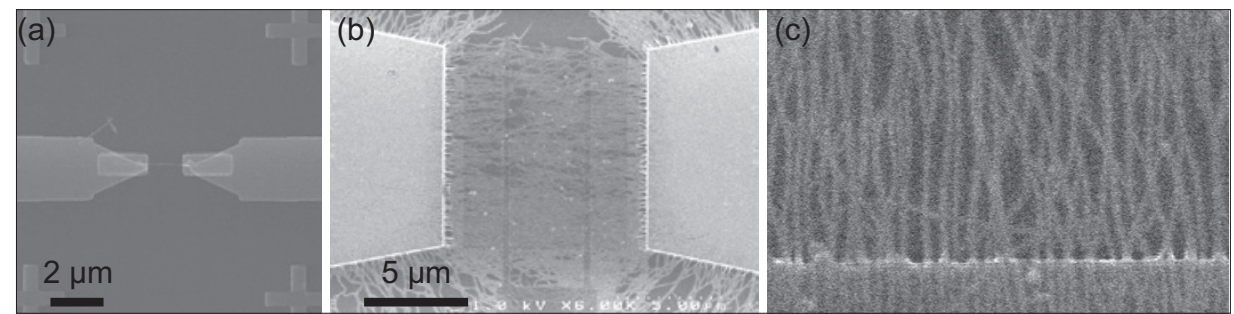

Fig. 3. Scanning electron microscopy (SEM) images of: (a) An individual MWNT selectively deposited by DEP from solution on predefined electrodes (and then re-contacted). (b) A dense network of SWNTs deposited by DEP between macroscopic electrodes and over a two-finger Al/ $\mathrm{AlO}_{x}$ back-gate electrode. This design is the one used (after source and drain contact fabrication) for the study of high frequency CNT transistors as described in the device section. ${ }^{[15]}$ (c) Higher magnification image showing the high degree of alignment of SWNTs deposited by DEP.

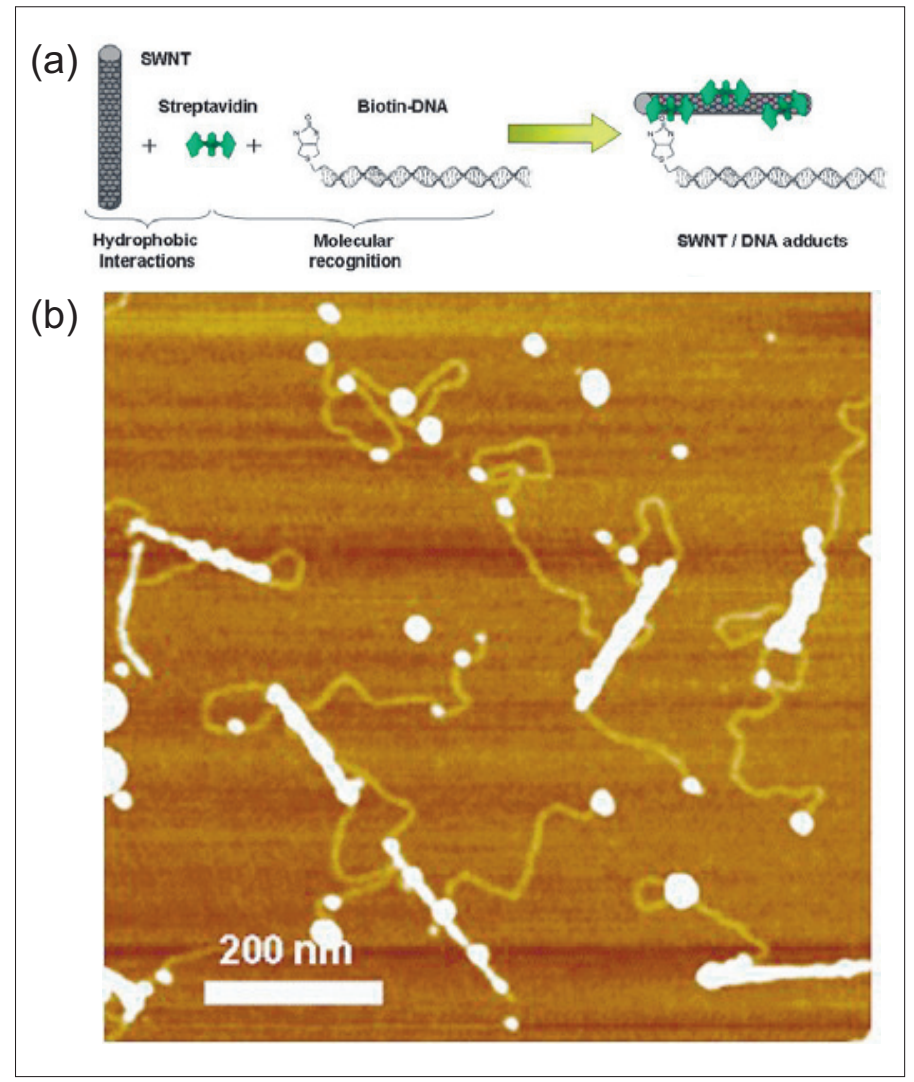

Fig. 4. (a) Principle of the non-covalent coupling of DNA to CNTs using the streptavidin-biotin recognition capabilities. (b) AFM images of CNT-DNA hybrids built by non-covalent coupling. After ref. [10].

biotin at tagged locations along DNA molecules $^{[10]}$ or close to the intersection site of T-shape DNA structures. ${ }^{[11]}$ Such synthesis of three branch DNA scaffolds and the insertion of CNTs at their centre are the first steps toward the bio-directed selfassembly of nanotube transistors with individual gates.

Once DNA-CNT hybrid objects have been prepared and deposited, the DNA scaffold can be transformed into an interconnected network by selective metallization of the DNA strands. ${ }^{[32,33]}$ To convert DNA into conducting leads, we developed a novel approach for DNA metallization. ${ }^{[34]}$ The progressive growth of nanowires was achieved by the slow and selective precipitation of palladium oxide on DNA molecules previously deposited on a dry substrate. The second step consisted on the reduction of the palladium oxide into metallic palladium. We fabricated homogeneous, continuous and conductive DNA-based Pd nanowires with very thin diameter (20-25 $\mathrm{nm}$ ). In addition, the proposed method is very selective as almost no parasitic metallization of the surface is obtained.

Once carbon nanotubes have been purified, solubilized, possibly functionalized and finally deposited by one of the selective deposition or self-assembly methods, they can be connected in a device geometry which allows the study and use of their electronic properties. A particularly interesting geometry is that of the field

\section{Versatile Use of SWNT-based Field Effect Transistors for New Functions and New Architectures}

effect transistor, in which a semiconducting nanotube serves as the channel, the conductivity of which is controlled by a capacitively coupled gate electrode. ${ }^{[35,36]}$ Due to the exceptional electronic properties of carbon nanotubes ${ }^{[37]}$ (in particular, the very long carrier mean free path, the high carrier velocity and the high current density capability), aggressively scaled carbon nanotube field effect transistors (CNTFETs) are among the best performing nano-scale FETs. ${ }^{[38-41]}$

At the LEM, we developed various type of CNTFETs and studied in particular their chemical sensitivity to minute modifications of their direct environment. ${ }^{[12,13]}$

\subsection{CNTFETs with a Molecular Gate Insulator}

Most of the CNTFETs reported in the literature were fabricated using inorganic dielectrics $\left(\mathrm{SiO}_{2}, \mathrm{Al}_{2} \mathrm{O}_{3}, \mathrm{HfO}_{2} \ldots\right)$. Recently, several groups proposed the use of organic or molecular ${ }^{[22,43]}$ dielectrics. It is an interesting step toward fully organic nano-scale transistors that could be fabricated on any type of substrate. It has also an important impact on performance. Indeed, most CNTFETs suffer from very severe hysteresis in their transfer characteristics $\left(\mathrm{I}_{\mathrm{D}}-\mathrm{V}_{\mathrm{GS}}\right)$, which principally originates from trap states in the inorganic dielectric layer and adsorbed molecules at their surface. Recently, we showed that a single molecular layer of alkanethiol molecules can be used as an ultra-thin organic dielectric for CNTFETs, as shown in the inset of Fig. 5. It consists of a single nanotube deposited on top of a thin gold electrode on which a self-assembled monolayer of octadecanethiol has been prepared. As shown in Fig. 5, no hysteresis is observed in the $\mathrm{I}_{\mathrm{D}}-\mathrm{V}_{\mathrm{GS}}$ characteristics due to the reduced density of trap states in such molecular layer and to its hydrophobic character. Moreover, the reduced dielectric thickness $(\sim 2.2 \mathrm{~nm})$ allows an ideal sub-threshold slope of $\sim 60 \mathrm{mV} / \mathrm{dec}$ to be achieved, proving the excellent gate coupling achieved in such configuration. ${ }^{[44]}$

\subsection{High-frequency CNTFETs}

Semiconducting SWNTs are one of the most promising materials for the development of electronic devices working at very high frequencies. Several theoretical estimations predict cut-off frequencies in the $\mathrm{THz}$ range for short channel nanotube transistors in the ballistic regime. ${ }^{[45,46]}$ More detailed models, in particular those developed at the IEF-Orsay in the group of P. Dollfus and S. Retailleau, include the impact of phonon scattering and have improved to a point where they become a valuable guide for the design of future high frequency devices. ${ }^{[47-49]}$

In close collaboration with the group of G. Dambrine at IEMN (CNRS, Lille), 


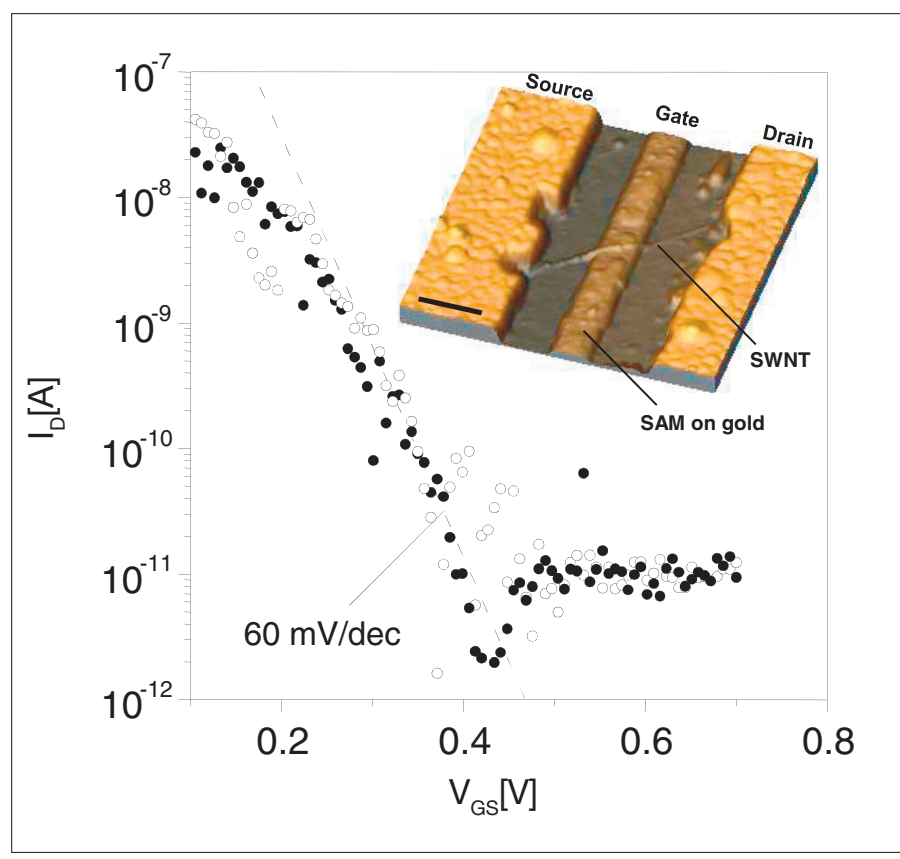

Fig. 5. Transfer characteristic $I_{D}\left(V_{G S}\right)$ of a carbon nanotube transistor using a self-assembled molecular mono-layer of octadecanethiol on gold as ultra-thin gate dielectric $\left(\mathrm{V}_{\mathrm{DS}}=\right.$ $-100 \mathrm{mV}$ ). The dotted line corresponds to the ideal $60 \mathrm{mV} / \mathrm{dec}$ subthreshold slope. Inset: AFM image illustrating the device geometry (adapted from ref. [44]).
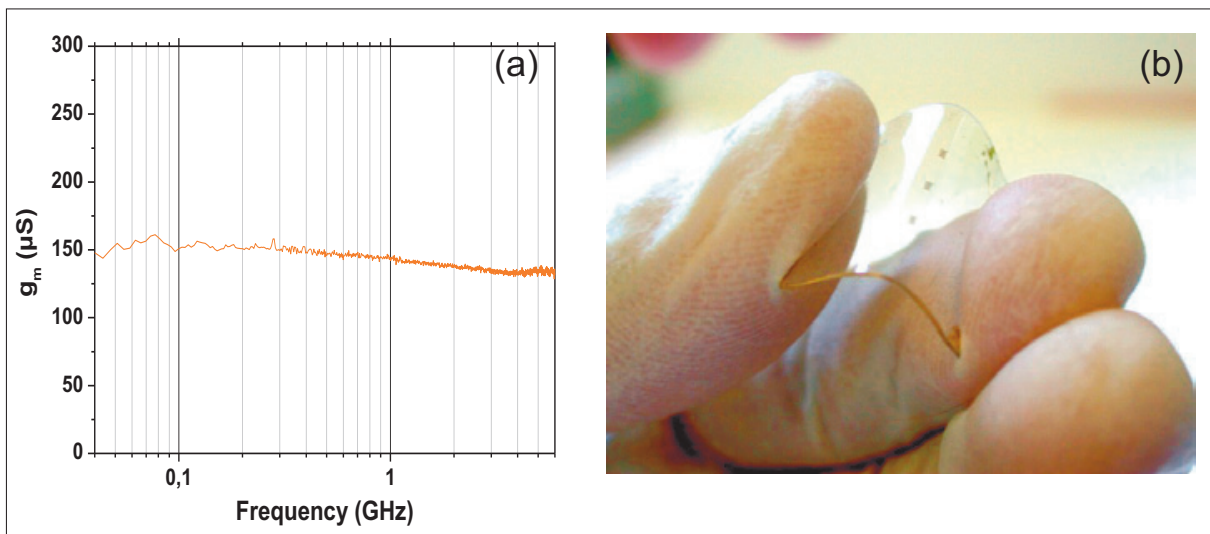

Fig. 6. (a) Frequency evolution of the flexible HF-CNTFET transconductance. (b) $3 \times 6 \mathrm{HF}$ nanotube transistors on a polyethylen-terephtalate film (PET). ${ }^{[16]}$

we developed high frequency carbon nanotube-based devices composed of multiple nanotubes in parallel, which allow a correct matching to $50 \Omega$ and lower the impact of parasitic capacitances. ${ }^{[14,15,50]}$ Benefiting from a collaboration with the group of M. Hersam at Northwestern University, that provides us with highly pure semiconducting nanotubes, we recently established the highest measured $\mathrm{f}_{\mathrm{T}}(80 \mathrm{GHz})$ for a nanotube-based device. ${ }^{[51]}$ However, it is still well below the intrinsic potential of nanotubes. Several routes for the improvement of these performances have been identified and are presently under investigation.

Interestingly, the methods of fabrication and characterization we used are compatible with any type of substrate, in particular plastics. This opens new opportunities for the realization of high frequency flexible electronic devices. Carbon nanotubes have been identified early as interesting candidates for the development of flexible electronic devices. ${ }^{[52-55]}$ Indeed, they combine the required mechanical flexibility with very high carrier mobility $\left(>10^{4} \mathrm{~cm}^{2} / \mathrm{V} . \mathrm{s}\right)$. This later advantage is particularly important when compared with conventional organic electronic materials such as semiconducting polymers, in which the carrier mobility is usually in the $1-10^{-3} \mathrm{~cm}^{2} / \mathrm{V}$.s range. Using the same strategy as for CNT devices on silicon, we recently demonstrated nanotube-based flexible transistors with as-measured $\mathrm{f}_{\mathrm{T}}$ as high as $1 \mathrm{GHz}^{[16]}$ and constant transconductance up to $6 \mathrm{GHz}$, as shown in Fig. 6 . We noticeably showed that the DC transconductance of such devices fabricated on a polyethylenterephtalate substrate remains constant down to radius of curvature of $\sim 3.3 \mathrm{~mm}$, a value already satisfactory for most envisioned flexible electronic applications. Thus, nanotubes prove by far more promising than conventional organic materials and already compete favourably with flexible semiconducting nanowires and ribbons. ${ }^{[56-58]}$ In this context, better performance at strong bending angles are expected due to the superior elasticity of CNTs.

\subsection{Optoelectronics Devices and New Architectures}

Semiconducting carbon nanotubes have also interesting optical properties including a direct band-gap, the energy of which depends on the nanotube diameter. From 2002, individual nanotubes have been incorporated into several types of optoelectronic devices, ${ }^{[59]}$ in particular nano-scale light sources ${ }^{[60]}$ and sensors.[61] Instead of relying on the optical properties of the nanotubes, which vary from one nanotube to the other, one can try to develop optoelectronic devices which combine the exceptional electrical properties of nanotubes with additional optical properties brought in by organic molecules. These molecules can be simply deposited or, better, chemically grafted on the nanotube (see part 2 and ref. [4]). Few examples of such strategy have been recently demonstrated. ${ }^{[19,62-65]}$ One particularly interesting route for both its simplicity and the originality of the achieved functionality consists in coating a CNTFET with a thin film of a photoconducting polymer. ${ }^{[19,62,64]}$ We fabricated CNTFETs using the chemical self-assembly technique described above and functionalized them with a thin film $(\sim 5 \mathrm{~nm})$ of poly (3-octyl-thiophene), a semiconducting conjugated polymer (OGCNTFET). We showed that the photo-generation of charges in the polymer allows the modulation of the nanotube conductivity over four orders of magnitude as presented in Fig. $7 b .^{[19]}$ In this configuration, the carbon nanotube acts as a local probe remarkably sensitive to the photo-generated charge distribution in the polymer film and at the polymer-dielectric interface and we proposed to use this probe to study organicinorganic interfaces that are important in the framework of organic electronics and solar cells. ${ }^{[20]}$

Moreover, the OGCNTFET is a nonvolatile, programmable memory. We recently showed that such optically-gated carbon nanotube transistors can be used as 2-terminal memory devices, i.e. programmable resistors, which have all the required characteristics to serve as building blocks for adaptive architectures. In particular, the nanotube channel resistivity can be precisely adjusted in a very large range spanning several orders of magnitudes and then stored in a non-volatile way, using source-drain bias pulses for programming. We also established the capability to handle the programming of multiple devices and demonstrated how this approach addresses the crucial issue of variability among devices. Altogether, it 

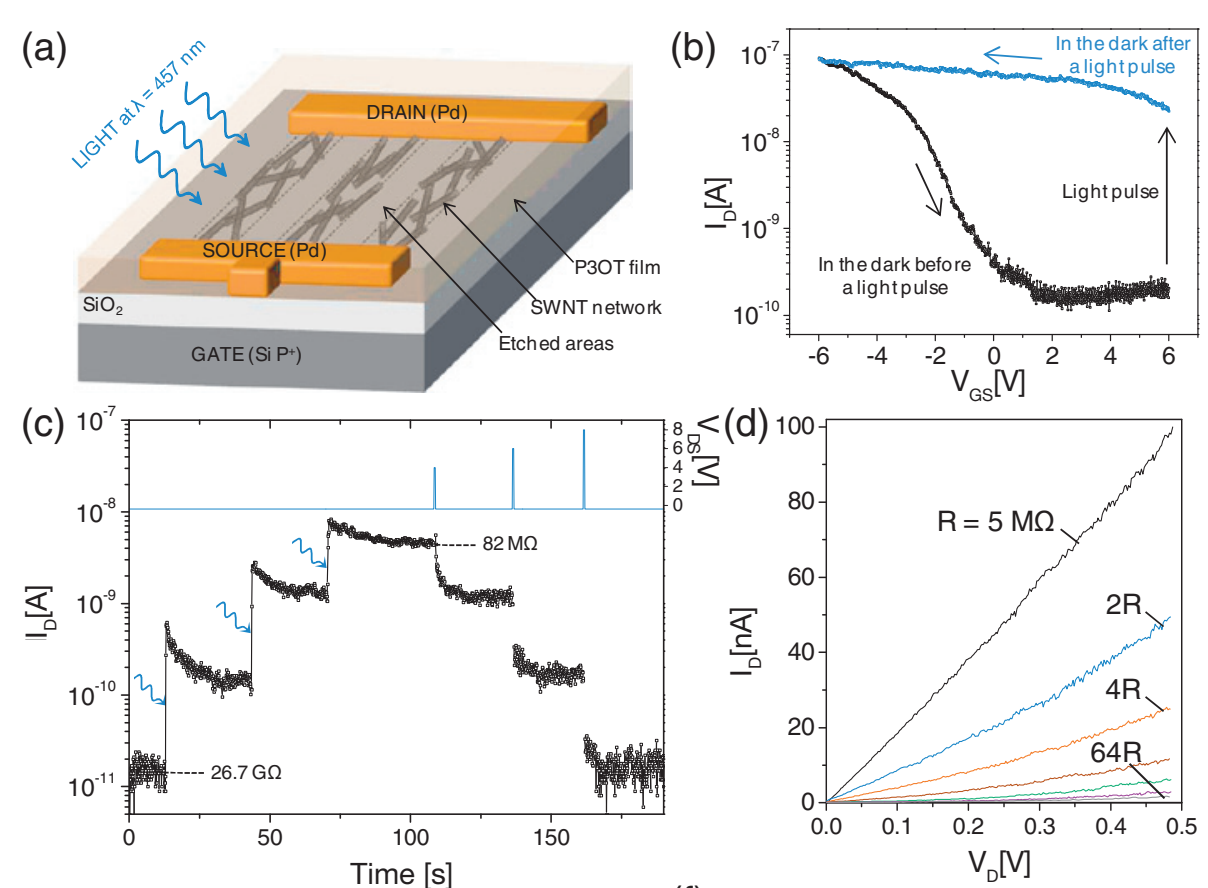

(e)

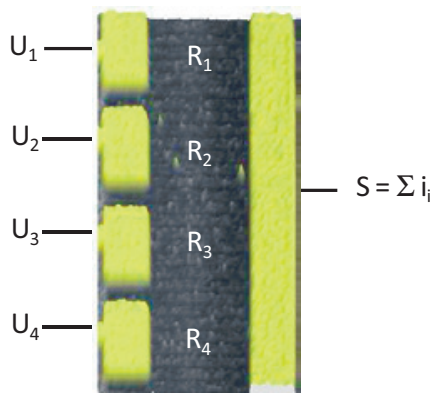

(f)
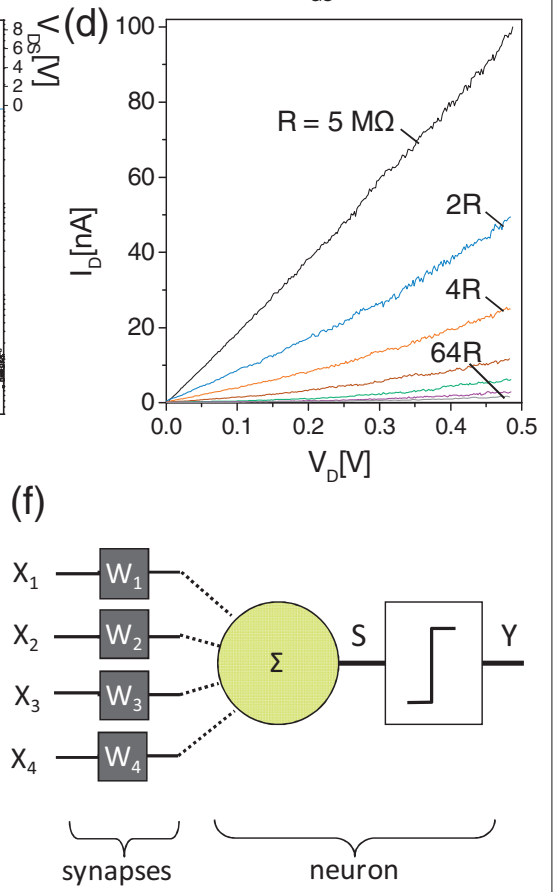

Fig. 7. (a) Schematic representation of a nanotube network-based OG-CNTFET. (b) Typical transfer characteristic $\mathrm{I}_{\mathrm{D}}\left(\mathrm{V}_{\mathrm{GS}}\right)$ of such OG-CNTFET (typical size $8 \mu \mathrm{m}$ long, 5 stripes of $500 \mathrm{~nm}$ width) in the dark before (black) and after (blue) a light pulse $\left(V_{D S}=-400 \mathrm{mV}\right)$. The arrows indicate the direction of the bias sweep. (c) Drain current as a function of time of an OG-CNTFET working as a 2-terminal device (at constant $\mathrm{V}_{\mathrm{GS}}=+2 \mathrm{~V}$ ). Light pulses and electrical pulses (applied on the source electrode) are used to program the resistivity of the device. (d) $I_{D}-V_{D S}$ curves acquired in the dark on the same OG-CNTFET at different programming stage (at constant $\mathrm{V}_{G S}=+3.5 \mathrm{~V}$ ). The programmed resistivity values are $R=5 \mathrm{M} \Omega, 2 R, 4 R, 8 R, 16 R, 32 R$ and 64R. (e) AFM image of a 4-inputs / 1-output circuit based on a stripes-structured network of SWNTs. The channel length is $8 \mu \mathrm{m}$. Each of the 4 devices is composed of 5 stripes of $500 \mathrm{~nm}$ in width. (f) Generic diagram of a perceptron. The resistivity of each device in (e) codes for the synaptic weights ( $\left.W_{\mathrm{i}}\right)$. (from ref. [21])

shows that such mixed nanotube-polymer devices have all the characteristics of artificial synapses that can provide an elegant solution to build neural network types of circuits (Fig. 7 and ref. [21]).

The presented devices could also play a role in other types of programmable architectures, such as FPGA (field-programmable gate array) where efficient and versatile non-volatile memories are also required.

\section{Conclusions}

In this review of our recent results, we showed that carbon nanotubes handled in solution can lead to the development of innovative electronic devices with re-

\section{Acknowledgments}

We acknowledge the important help of $\mathrm{P}$. Lavie (CEA), T. Torres (UAM Madrid), D. M. Guldi (Erlangen-Nürnberg University), M. Prato (Trieste University), O. Jost (TU Dresden), H. Happy, G. Dambrine, S. Lenfant, D. Vuillaume (CNRS-IEMN), B. Giffard, J.C. Gabriel, R. Baptist (CEA-LETI) and all the students and post-docs that participated to the work: G. Agnus, S. Auvray, J. Borghetti, C.L. Chung. R. Lefèvre, K. Nguyen, G. Robert, G. Schmidt, C. Anghel, N. Chimot, S. Lyonnais, S. Streiff. This work was partially funded by the ANR through the projects BIO-NT, ACCENT, HF-CNT, MEMO, PIXEL and PANINI, and by the E.U through the projects Nano-RF, NUCAN, NABAB and Chemtronics. We also acknowledge the financial support of the C'Nano IdF (project GHZ-NT), the Région Ile-de-France (Sesame project), the GdR Nanotubes and the CEA Chimtronique program.

Received: April 29, 2010

[1] 'Nanoscience Nanotechnologies and Nanophysics', Eds. C. Dupas, P. Houdy, M. Lahmani, 2007, ISBN 978-3-540-28616.

[2] L. Capes, E. Valentin, S. Esnouf, A. Ribayrol, O. Jost, A. Filoramo, J. N. Patillon, in 'Proc. IEEE Conf. on Nanotechnology', 2002, pp 439.

[3] G. Schmidt, S. Gallon, S. Esnouf, J.-P. Bourgoin, P. Chenevier, Chem. Eur. J. 2009, 15, 2101.

[4] S. Campidelli, B. Ballesteros, A. Filoramo, D. Díaz Díaz, G. de la Torre, T. Torres, G. M. A. Rahman, C. Ehli, D. Kiessling, F. Werner, V. Sgobba, D. M. Guldi, C. Cioffi, M. Prato, J.-P. Bourgoin, J. Am. Chem. Soc. 2008, 130, 11503.

[5] K. H. Choi, J.-P. Bourgoin, S. Auvray, D. Estève, G.S. Duesberg, S. Roth, M. Burghard, Surf. Sci. 2000, 472, 195.

[6] E. Valentin, S. Auvray, J. Goethals, J. Lewenstein, L. Capes, A. Filoramo, A. Ribayrol, R. Tsui, J-P. Bourgoin, J-N. Patillon, Microelect. Eng. 2002, 61, 491.

[7] E. Valentin, S. Auvray, A. Filoramo, A. Ribayrol, M. F. Goffman, L. Capes, J.-P. Bourgoin, J.-N Patillon, Proc. MRS Symp. 2003, 772, 201.

[8] E. Valentin, S. Auvray, A. Filoramo, A Ribayrol, M. F. Goffman, J. Goethals, L. Capes, J.-P. Bourgoin, J.-N. Patillon, in 'Proc. of the NATO Advanced Research Workshop on Molecular Nanowires and Other Quantum Objects', Eds. A. S. Alexandrov, J. Demsar, I. K. Yanson, Vol. 148 of NATO Science Series II Springer-Verlag, Berlin, 2004, pp 57.

[9] C.-L. Chung, K. Nguyen, S. Lyonnais, S. Streiff, S. Campidelli, L. Goux-Capes, J.-P. Bourgoin, A. Filoramo, in 'AIP Proc. of the Int. Symp. on DNA-Based Nanodevices' Jena, Germany, 29-31 May 2008, ISBN 978-0-7354-0592-9.

[10] S. Lyonnais, L. Goux-Capes, C. Escudé, D. Cote, A. Filoramo, J.-P. Bourgoin, Small 2008, $4,442$.

[11] S. Lyonnais, C-L. Chung, L. Goux-Capes, C. Escudé, O. Piétrement, S. Baconnais, E. Le Cam, J.-P. Bourgoin, A. Filoramo, Chem. Commun. 2009, 683

[12] S. Auvray, J. Borghetti, M.F. Goffman, A Filoramo, V. Derycke, J.-P. Bourgoin, O. Jost, App. Phys. Lett. 2004, 84, 5106.

[13] S. Auvray, A. Filoramo, M. F. Goffman, V. Derycke, O. Jost, J.-P. Bourgoin, Nano Lett. 2005, 5,451 .

[14] J.-M. Bethoux, H. Happy, G. Dambrine, V Derycke, M. F. Goffman, J.-P. Bourgoin, IEEE Electron Device Lett. 2006, 27, 681.

[15] A. Le Louarn, F. Kapche, J.-M. Bethoux, H. Happy, G. Dambrine, V. Derycke, P. Chenevier, 
N. Izard, M. F. Goffman, J.-P. Bourgoin, Appl. Phys. Lett. 2007, 90, 233108.

[16] N. Chimot, V. Derycke, M. F. Goffman, J. P. Bourgoin, H. Happy, G. Dambrine, Appl. Phys. Lett. 2007, 91, 53111.

[17] R. Lefèvre, M. F. Goffman, V. Derycke, C. Miko, L. Forr'o, J.-P. Bourgoin, P. Hesto, Phys. Rev. Lett. 2005, 95, 185504.

[18] E. Dujardin, V. Derycke, M. F. Goffman, R. Lefèvre, J.-P. Bourgoin, Appl. Phys. Lett. 2005, 87, 193107.

[19] J. Borghetti, V. Derycke, S. Lenfant, P. Chenevier, A. Filoramo, M. F. Goffman, D. Vuillaume, J.-P. Bourgoin, Adv. Mater. 2006, $18,2535$.

[20] C. Anghel, V. Derycke, A. Filoramo, S. Lenfant, B. Giffard, D. Vuillaume, J.-P. Bourgoin, Nano Lett. 2008, 8, 3619.

[21] G. Agnus, W. Zhao, V. Derycke, A. Filoramo, Y. Lhuillier, S. Lenfant, D. Vuillaume, C. Gamrat, J.-P. Bourgoin, Adv. Mater. 2010, 22, 702.

[22] H. C. Kolb, M. G. Finn, K. B. Sharpless, Angew. Chem. Int. Ed. 2001, 40, 2004.

[23] R. Huisgen, in '1,3-dipolar Cycloaddition Chemistry', Ed. A. Padwa, Wiley, New York, 1984, pp 1.

[24] V. V. Rostovtsev, L. G. Green, V. V. Fokin, K. B. Sharpless, Angew. Chem. Int. Ed. 2002, 41, 2596.

[25] C. W. Tornøe, C. Christensen, M. Meldal, J. Org. Chem. 2002, 67, 3057.

[26] V. D. Bock, H. Hiemstra, J. H. van Maarseveen, Eur. J. Org. Chem. 2006, 1, 51.

[27] H. Li, F. Cheng, A. M. Duft, A. Adronov, J. Am. Chem. Soc. 2005, 127, 14518.

[28] Z. Guo, L. Liang, J.-J. Liang, Y.-F. Ma, X.-Y. Yang, D.-M. Ren, Y.-S. Chen, J.-Y. Zheng, J. Nanopart. Res. 2008, 10, 1077.

[29] J. Liu, M. J. Casavant, M. Cox, D. A. Walters, P. Boul, W. Lu, A. J. Rimberg, K. A. Smith, D. T. Colbert, R. E. Smalley, Chem. Phys. Lett. 1999, $303,125$.

[30] J. Muster, M. Burghard, S. Roth, G. S. Duesberg, E. Hernández, A. Rubio, J. Vac. Sci. Tech. B 1998, 16, 2796.

[31] R. Krupke, F. Hennrich, H. von Lohneysen, M. M. Kappes, Science 2003, 301, 344.

[32] N. C. Seeman, Nature 2003, 421, 427.

[33] J. Richter, Physica E 2003, 16, 157.
[34] K. Nguyen, M. Monteverde, A. Filoramo, L. Goux-Capes, S. Lyonnais, P. Jegou, P. Viel, M. Goffman, J.-P. Bourgoin, Adv. Mater. 2008, 20, 1099.

[35] S. J. Tans, A. R. M. Verschueren, C. Dekker, Nature 1998, 393, 49.

[36] R. Martel, T. Schmidt, H. R. Shea, T. Hertel, P. Avouris, Appl. Phys. Lett. 1998, 73, 2447.

[37] J. C. Charlier, X. Blase, S. Roche, Rev. Mod. Phys. 2007, 79, 677.

[38] A. Javey, J. Guo, D. B. Farmer, Q. Wang, E. Yenilmez, R. G. Gordon, M. Lundstrom, H. Dai, Nano Lett. 2004, 4, 1319.

[39] R. V. Seidel, A. P. Graham, J. Kretz, B. Rajasekharan, G. S. Duesberg, M. Liebau, E. Unger, F. Kreupl, W. Hoenlein, Nano Lett. 2005, 5, 147.

[40] A. Javey, P. Qi, Q. Wang, H. Dai, Proc. Natl. Acad. Sci. USA 2004, 101, 13408.

[41] Y. M. Lin, J. Appenzeller, Z. H. Chen, Z. G. Chen, H. M. Cheng, P. Avouris, IEEE Elec. Dev. Lett. 2005, 26, 823.

[42] S-H. Hur, M-H. Yoon, A. Gaur, M. Shim, A. Facchetti, T. J. Marks, J. A. Rogers, J. Am. Chem. Soc. 2005, 127, 13808.

[43] R. T. Weitz, U. Zschieschang, F. Effenberger, H. Klauk, M. Burghard, K. Kern, Nano Lett. 2007, $7,22$.

[44] G. Robert, V. Derycke, M. F. Goffman, S Lenfant, D. Vuillaume, J-P Bourgoin, Appl. Phys. Lett. 2008, 93, 143117.

[45] P. J. Burke, Solid-State Electronics 2004, 48 , 1981.

[46] J. Guo, S. Hasan, A. Javey, G. Bosman, M. Lundstrom, IEEE Trans. on. Nanotechnol. 2005, 4,715 .

[47] H. Cazin d'Honincthun, S. Galdin-Retailleau, A. Bournel, P. Dollfus, J-P. Bourgoin, C. $R$. Physique 2008, 9, 67.

[48] H. Cazin d'Honincthun, H. N. Nguyen, S. Galdin-Retailleau, A. Bournel, P. Dollfus, J.-P. Bourgoin, Phys. E Low Dim. Sys. \& Nanostruct. 2008, 40, 2294

[49] S. Fregonese, H. Cazin d'Honincthun, J. Goguet, J. Maneux, T. Zimmer, J.-P. Bourgoin, P. Dollfus, S. Galdin-Retailleau, IEEE Trans. on Elec. Dev. 2008, 55, 1317.

[50] J-M. Bethoux, H. Happy, A. Siligaris, G. Dambrine, J. Borghetti, V. Derycke, J-P
Bourgoin, IEEE Trans on Nanotechnol. 2006, 5,335 .

[51] L. Nougaret, H. Happy, G. Dambrine, 1 V. Derycke, J. -P. Bourgoin, A. A. Green, M. C. Hersam, Appl. Phys. Lett. 2009, 94, 243505.

[52] K. Bradley, J. C. P. Gabriel, G. Gruner, Nano Lett. 2003, 3, 1353.

[53] E. Artukovic, M. Kaempgen, D. S. Hecht, S. Roth, G. Gruner, Nano Lett. 2005, 5, 757.

[54] Q. Cao, S. H. Hur, Z. T. Zhu, Y. Sun, C. Wang, M. A. Meitl, M. Shim, J. A. Rogers, Adv. Mater. 2006, 18, 304.

[55] S. J. Kang, C. Kocabas, T. Ozel, M. Shim, N. Pimparkar, M. A. Alam, S. V. Rotkin, J. A. Rogers, Nat. Nanotechnol. 2007, 2, 230.

[56] H. C. Yuan, Z. Ma, Appl. Phys. Lett. 2006, 89, 212105.

[57] J. H. Ahn, H. S. Kim, K. J. Lee, Z. Zhu, E. Menard, R. G. Nuzzo, J. A. Rogers, IEEE Electron. Dev. Lett. 2006, 27, 460.

[58] Y. Sun, E. Menard, J. A. Rogers, H. S. Kim, S. Kim, G. Chen, I. Adesida, R. Dettmer, R. Cortez, A. Tewksbury, Appl. Phys. Lett. 2006, 88, 183509.

[59] P. Avouris, M. Freitag, V. Perebeinos, Topics in Appl. Phys. 2008, 111, 423.

[60] J. A. Misewich, R. Martel, P. Avouris, J. C. Tsang, S. Heinze, J. Tersoff, Science 2003, 300, 783.

[61] M. Freitag, Y. Martin, J. A. Misewich, R. Martel, P. Avouris, Nano Lett. 2003, 3, 1067.

[62] A. Star, L. Yu, K. Bradley, G. Gruner, Nano Lett. 2004, 4, 1587.

[63] X. Guo, L. Huang, S. O'Brien, P. Kim, C. Nuckolls, J. Am. Chem. Soc. 2005, 127, 15045.

[64] K. S. Narayan, M. Rao, R. Zhang, P. Maniar, Appl. Phys. Lett. 2006, 88, 243507.

[65] J. M. Simmons, I. In, V. E. Campbell, T. J. Mark, F. Léonard, P. Gopalan, M. A. Eriksson, Phys. Rev. Lett. 2007, 98, 086802.

[66] V. Derycke, S. Auvray, J. Borghetti, C. L. Chung, R. Lefevre, A. Lopez-Bezanilla, K. Nguyen, G. Robert, G. Schmidt, C. Anghel, N. Chimot, S. Latil, X. Blase, F. Triozon, S. Roche, J.P. Bourgoin, C. R. Physique 2009, 10, 330. 